\title{
54. On the Chromosomes of the Wild Boar and the Boar-Pig Hybrids ${ }^{11}$
}

\author{
By Jun-ichi Muramoto, ${ }^{*)}$ Sajiro Makino, ${ }^{*)}$ Tsune Ishikawa, $\left.{ }^{* *}\right)$ \\ and Hiroshi Kanagawa**) \\ (Comm. by Yoshimaro TANAKA, M.J.A., March 12, 1965)
}

Since the latter half of the 18th century, the evolutional relationship between the pig and the wild boar has attracted attention of many investigators. The literature pertaining to this problem refers to many studies mostly based on morphological and serological standpoints. Makino (1945) is a sole author who dealt with the relationship of chromosomes between the two species based on the classic paraffin method. At the present status of increased cytogenetic knowledge in mammals, need is growing for a reinvestigation on the chromosomes of these species through the application of improved techniques. On the basis of the above view, Makino and his co-workers have undertaken reinvestigations of the chromosomes of domestic mammals (Awa et al. 1959, Nakanishi and Mizutani 1959, Nakanishi 1960, Sasaki and Makino 1962, Sasaki and Sasaki 1962, Makino et al. 1962, 1963). This report gives in some details the chromosome features of the wild boar (Sus vittatus leucomystax Major), and hybrids between the wild boar and the pig (Sus scrofa) by the application of current tissue culture techniques.

The materials for this study were supplied by Mr. S. Matsuo and Mr. S. Itô, the Takikawa Animal Husbandry Experiment Station, to whom the authors are much grateful.

Material and Method: A female wild boar (Sus vittatus leucomystax Major) and the hybrids between the wild boar (우) and the pig (令) (Sus scrofa) have been bred in the Takikawa Animal Husbandry Experiment Station, Hokkaido. The wild boar aged 11years, and 5-month-old hybrids of both sexes, provided the materials for this study. Chromosomal data were exclusively derived from leucocyte cultures. The technique applied for the study is as follows: The supernatant from heparinized venous blood stored in the ice box for about 3 hours was mixed with culture medium at a proportion. of $1: 8$ with the addition of phytohemagglutinin- $\mathrm{M}(0.2 \mathrm{ml} / 10 \mathrm{ml}$ of medium). The mixture was transferred into TD-15 flasks and incu-

1) Contribution No. 681 from the Zoological Institute, Faculty of Science, Hokkaido University, Sapporo. Supported by a grant-in-aid for Scientific Research from the Ministry of Education (1965).

*) Zoological Institute, Hokkaido University.

**) Faculty of Veterinary Science, Hokkaido University. 
bated at $37^{\circ} \mathrm{C}$. After 85 hours of incubation, mitotic cells were arrested by colchicine treatment $(0.01 \mathrm{ml} / 1 \mathrm{ml})$ for 1 to 2 hours. After water-pretreatment for 15 minutes, slides for chromosome study were made according to the air-drying technique. Details of the method will be given in another paper.

Results: The chromosome number of the wild boar was determined as $2 n 38$, on the basis of data shown in Table I (Figs. 1-2). The same diploid number was established in the pig by Makino et al. (1962). They tentatively classified the chromosomes into 7 groups. Comparison of chromosomes between the pig and the wild boar revealed no appreciable difference (Figs. 1-3). Therefore, the following descriptions can be applied to both.

Table I. Results of chromosome counts in the wild boar and the boar-pig hybrids

\begin{tabular}{lcrrrr}
\hline \multirow{2}{*}{ Case } & \multirow{2}{*}{ Material } & \multicolumn{3}{c}{ Chrom. counts } & \multirow{2}{*}{ Total cells } \\
\cline { 3 - 4 } & & 37 & 38 & 39 & obs. \\
\hline Female wild boar & Leucocyte & 2 & 50 & 2 & 54 \\
Female hybrid & $\prime \prime$ & 2 & 54 & & 56 \\
Male hybrid & $\prime \prime$ & 1 & 53 & 1 & 55 \\
\hline
\end{tabular}

The results of karyotype analyses in both species are described as follows: Group 1 contains a pair of the largest submetacentric chromosomes. Group 2 consists of 16 metacentric and submetacentric chromosomes of almost similar size. It is especially noted that chromosomes of the 8th pair carry a remarkable secondary constriction. Group 3 is represented by a pair of chromosomes also carrying in each a consistent and well-defined secondary constriction. In wellspread cells, each of the chromosomes no. 10 (group 3) looks like two apart separate entities. The situation occurring in a pair of group 3 (chromosomes no. 10) apparently led to a miscount of the chromosome number in the classical sectioned materialo (Makino 1944). Group 4 includes a pair of acrocentric chromosomes. Four acrocentric chromosomes of nearly similar size are categorized in group 5. They are apparently smaller than those of group 4. Group 6 contains three pairs of acrocentric chromosomes. Group 7 comprises two pairs of metacentric chromosomes, almost similar in morphology. The $\mathrm{X}$ chromosome corresponds in size to the chromosomes belonging to group 2. The $\mathrm{Y}$ chromosome is clearly distinguishable from the others on account of its smallest size.

The chromosome number in the boar-pig hybrids was determined as 38 in the diploid complements of both sexes (Figs. 4-5, Table I). It is thus evident that the chromosome number of the hybrids is essentially identical with those of the female and male pigs (Makino 
et al. 1962; Gimenez-Martin et al. 1962; Ruddle 1964).

As shown in Table I, the vast majority of cells counted in the wild boar, and in the boar-pig hybrids showed the same chromosome number, 38. Further, morphologically the chromosome complement of the female hybrid is also consistently identical with that of the female wild boar (Figs. 1-2,4). The $\mathrm{Y}$ is represented by the smallest metacentric chromosome, being clearly distinguishable from the other
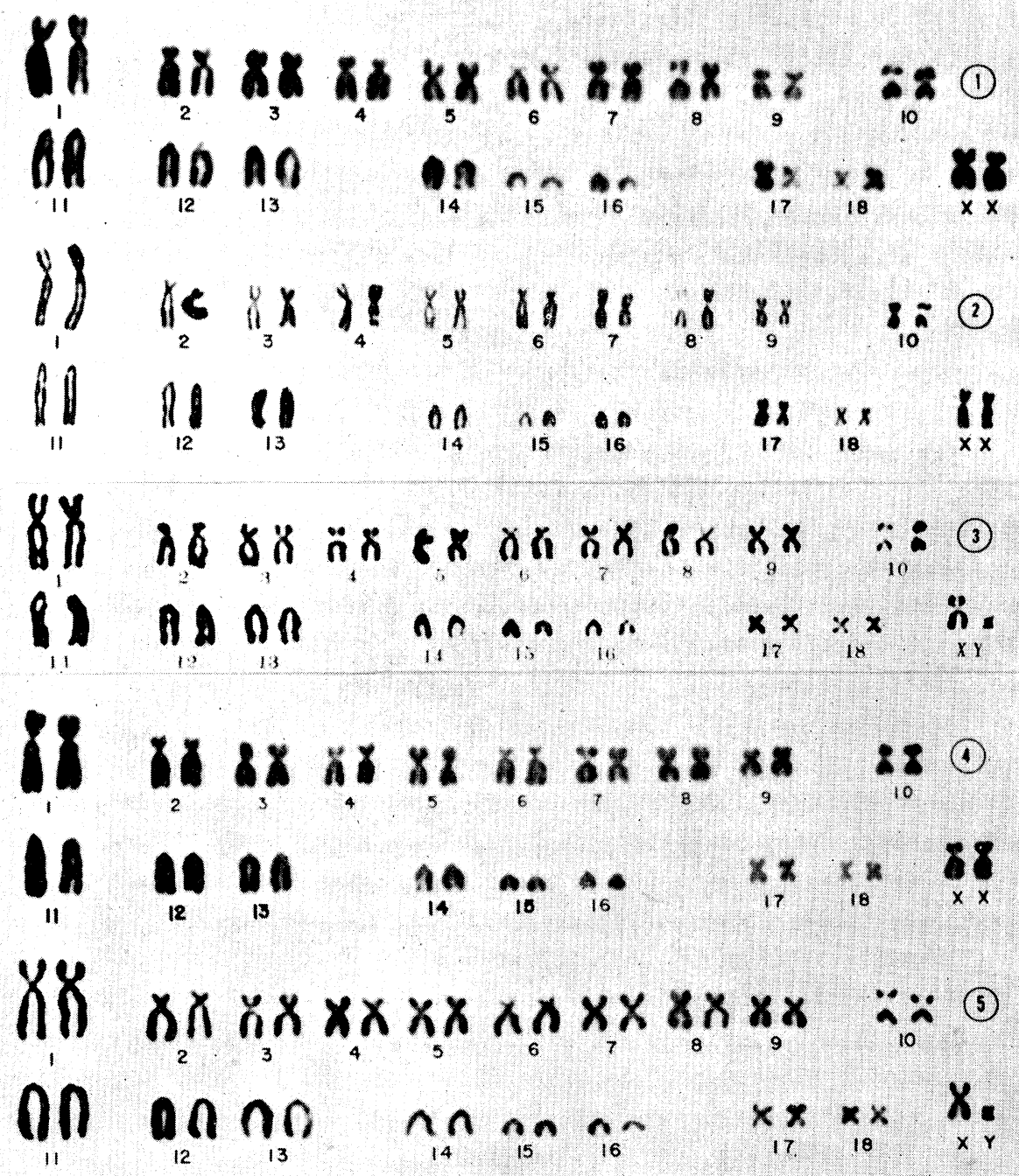

Figs. 1-5. Karyotypes of a wild boar, a pig and the boar-pig hybrids. Figs. 1-2. Karyotypes of cells from the female wild boar. Fig. 3. Karyotype of a cell from the male pig. Figs. 4 and 5. Karyotype analyses in a female hybrid (4), and in a male hybrid (5). 
elements. The $\mathrm{X}$ was found to be the same as the $\mathrm{X}$ of the pig. A karyotype from a male pig is shown in Fig. 3 for comparison.

Remarks: The present investigation established that the chromosome complements of the pig and the boar-pig hybrids were essentially identical both numerically and morphologically, showing the diploid number of 38. It is quite natural to interpret that a half set of the hybrid chromosome complement originated from a female wild boar and the other set from a male pig. Apparently the hybrids would receive 19 chromosomes from both parents, 18 autosomes and an $\mathrm{X}$ from the wild boar and the same set of autosomes and a $\mathrm{Y}$ or an $\mathrm{X}$ from the pig. Cytological evidence obtained suggests a close kinship between the boar and the pig, as well as the fertility of the hybrids.

Makino (1946) reported that the chromosome number of the wild boar as well as of the pig was $2 n 40$, based on the classical sectioned material. The mistake in chromosome count is attributable to the existence of well-defined secondary constrictions occurring in two homologous chromosomes belonging to the no. 10th pair (group 3). Miscount in the chromosome number of the pig as reported by some previous authors seems also to be ascribable to similar cause (Hance 1918, Makino 1944, Sachs 1954). The results of recent investigations based on the tissue culture method are in good agreement in showing that the correct number of the pig is 38 in $2 n$ (Makino et al. 1962; Gimenez-Martin et al. 1962; Ruddle 1964). Further details will be given by the senior author in another paper.

Summary: It was observed on the basis of leucocyte cultures that the somatic chromosome number of the wild boar (Sus vittatus leucomystax Major) and the boar-pig hybrids was 38 . The chromosome complements of the wild boar, the pig and the boar-pig hybrids were essentially identical with each other both numerically and morphologically. Cytological evidence suggests the fertility of the hybrids.

\section{References}

Awa, A., M. Sasaki, and S. Takayama (1959): Jap. Jour. Zool., 12, 257-265.

Gimenez-Martin, G., J. F. Lopez-Saez, and E. G. Monge (1962): J. Hered., 53, 281.

Hance, R. T. (1917): J. Morph., 30.

Makino, S. (1944): Cytologia, 13, 170-178.

- (1946): La Kromosomo, 1, 12-17.

- (1955): Experientia, II, 224-229.

Makino, S., M. S. Sasaki, and T. Sofuni (1962): Proc. Japan Acad., 38, 686-689. Makino, S., T. Sofuni, and M. S. Sasaki (1963): Proc. Japan Acad., 39, 176-181. Nakanishi, Y. H., and M. Mizutani (1959): Texas Rep. Biol. Med., 17, 134-141. Nakanishi, Y. H. (1960): Zeit. Zellforsch., 51, 138-151.

Ruddle, F. H. (1964): Symp. Internat. Soc. Cell Biol., 3, 157-162.

Sasaki, M. S., and S. Makino (1962): J. Hered., 53, 157-162.

Sasaki, M. S., and M. Sasaki (1962): Cytogenetics, 1, 291-300. 